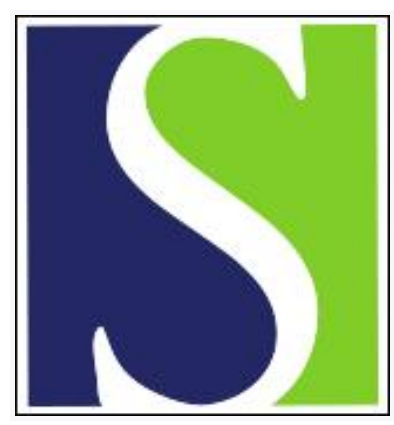

Scand J Work Environ Health 1996;22(3):204-210

https://doi.org/10.5271/sjweh.132

Issue date: Jun 1996

Vibrotactile perception thresholds as determined by two different devices in a working population

by Wenemark M, Lundström R, Hagberg M, Nilsson T

Key terms: hand; neuropathy; neurophysiology; occupational health; tactile perception; vibration

This article in PubMed: www.ncbi.nlm.nih.gov/pubmed/8837266

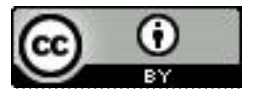




\title{
Vibrotactile perception thresholds as determined by two different devices in a working population
}

\author{
by Marika Wenemark, BSc, ${ }^{1}$ Ronnie Lundström, DrMedSc, ${ }^{2}$ Mats Hagberg, MD, ${ }^{1}$ Tohr Nilsson, MD ${ }^{3}$
}

\begin{abstract}
Wenemark M, Lundström R, Hagberg M, Nilsson T. Vibrotactile perception thresholds as determined by two different devices in a working population. Scand J Work Environ Health 1996;22:204-10.

Objectives The purpose of this study was to investigate whether two devices for measuring vibrotactile perception thresholds produced similar results on an individual basis and to compare the thresholds in the presence or absence of sensorineural hand symptoms and vibration exposure.

Methods Vibrotactile perception thresholds were measured with a vibrameter and a tactilometer in $178 \mathrm{men}$. The tactilometer uses seven discrete frequencies from 8 to $500 \mathrm{~Hz}$, and the vibrameter uses one frequency $(100 \mathrm{~Hz})$. Agreement was assessed from the correlations and from a comparison of subjects who had thresholds above the upper quartile of each device as to the presence of sensorineural hand symptoms and occupational vibration exposure.

Results The correlation between the vibrameter and the tactilometer $(125 \mathrm{~Hz})$ was 0.59 . The agreement between the vibrameter and the tactilometer $(63 \mathrm{~Hz})$, when the upper quartile was used as a limit, had a sensitivity of 0.56 and a specificity of 0.85 . The sensitivity and specificity for the agreement with symptoms were 0.44 and 0.79 , respectively, for the tactilometer and 0.40 and 0.78 , respectively, for the vibrameter. The indices combined from the different frequencies of the tactilometer did not improve the agreement.

Conclusions Some of the discrepancy between the measurement of the vibrameter and tactilometer can be explained by differences in the equipment, the measurement procedures, and the examiner, combined with high inter- and intraindividual variability. Neither of the two devices was superior when the results were compared as to the occurrence of hand symptoms and vibration exposure. As evaluated in this study vibrotactile perception threshold has a restricted value for screening and diagnostic purposes on an individual basis.
\end{abstract}

Key terms hand, neuropathy, neurophysiology, occupational health, tactile perception, vibration.

Vibrotactile perception thresholds (VPT) are becoming increasingly popular as a diagnostic tool for the early detection of vibration-induced neuropathy $(1-4)$. They have also been suggested as a possible method for the sensorineural disturbance staging proposed at the 1986 Stockholm Workshop (5). VPT can be determined by several different devices, such as the Somedic vibrameter (Stockholm, Sweden), the Brüel \& Kjaer vibrometer (Naerum, Denmark), the VibroMedic tactilometer (Malmö, Sweden), the biothesiometer of Biomedical Instruments (Newbury, Ohio, United States), the Vibraton II ${ }^{(1)}$ of Physitemp (Clifton, New Jersey, United States) and the Optacon ${ }^{\circledast}$ of Telesensory Systems (Palo Alto, California, United States). The devices have differences that are known to influence the threshold, such as the frequency of vibration, the diameter of the vibrating probe, the method of assessing the threshold, and the contact force between the probe and the finger $(6,7)$. The thresholds are also influenced by individual factors such as age, thickness of the skin, and skin temperature $(6,8,9)$. It is not known, however, if two devices can produce a similar classification between normal and abnormal vibrotactile perception in spite of the differences. This study concerned VPT measurements determined by two devices, the Somedic vibrameter, which measures at a single frequency $(100 \mathrm{~Hz})$, and the Brüel \& Kjaer tactilometer, which produces a tactilogram consisting of thresholds at seven discrete frequencies from 8 to $500 \mathrm{~Hz}$. The objective was to compare the vibrotactile perception thresholds determined by the two devices and to compare the thresholds with the presence of sensorineural hand symptoms and occupational vibration exposure.

1 National Institute of Occupational Health, Division of Work and Environmental Physiology, Solna, Sweden.

2 National Institute of Occupational Health, Division of Technical Industrial Hygiene, Umeå, Sweden.

3 Sundsvall County Hospital, Department of Occupational Medicine, Sundsvall, Sweden.

Reprint requests to: Dr Ronnie Lundström, National Institute of Occupational Health, Division of Technical Industrial Hygiene, PO Box 7654, S-907 13 Umeå, Sweden. 


\section{Subjects and methods}

\section{Subjects}

The study comprised 178 men employed as engineering workers and office workers at a heavy engineering production workshop in Sweden (table 1). The participants spent around $2 \mathrm{~h}$ answering a questionnaire, doing different functional tests and being interviewed and examined by a physician following a prewritten protocol. The questionnaire contained questions about basic information such as age, work assignments, general state of health, years at work, and so forth. It also contained the following inquiry about symptoms of sensorineural disturbances in the hands: "Do you currently have the following symptoms: nocturnal numbness in hands or fingers, trouble with dropping things easily, difficulties buttoning?"

The answers were given on a four-grade scale (no, insignificant, some, rather a lot) for the right and left hands separately. In the analysis, the subjects who had marked the alternatives "some" or "rather a lot" for any of the three symptoms were classified as having sensorineural hand symptoms.

Employment history was established from the employment lists at the workplace, from the results of the questionnaire, and, in some cases, from an interview about previous occupations and workplaces. If a subject had ever worked in a profession in which the use of vibrating handheld tools were involved, he was classified as exposed. Work with vibrating handheld tools during spare time was not taken into consideration.

\section{Apparatus and experimental procedure}

The minimum occupational vibration-free time before the measurements was at least $15 \mathrm{~h}$. An exposure-free time of several hours has been recommended for the study of chronic effects (10). After finishing one of the functional tests, the subject continued to another that was not in use at that moment. The order of the two VPT procedures was therefore not specified. The time between the two VPT procedures was approximately $30 \mathrm{~min}$.

The VPT measurements were conducted bilaterally on the pulp of the index finger. Altogether 178 right hands and 173 left hands were examined. The five men did not participate with the left hand because they had a finger missing or there was some physical injury on the finger, such as a cut or scar. For both instruments the subject sat with the test arm resting on a support. One well-experienced examiner for each instrument conducted the measurements for all the subjects. The skin temperature of the finger was checked before the measurements. If the temperature was below $28^{\circ} \mathrm{C}$ it was
Table 1. Age and frequency of symptoms of the group exposed to vibration and the group not exposed to vibration.

\begin{tabular}{|c|c|c|c|c|c|}
\hline \multirow[t]{2}{*}{ Group } & \multirow{2}{*}{$\begin{array}{l}\text { Number } \\
\text { of } \\
\text { workers } \\
\text { (N) }\end{array}$} & \multicolumn{2}{|c|}{ Age } & \multicolumn{2}{|c|}{$\begin{array}{c}\text { Sensorineural } \\
\text { hand symptoms }\end{array}$} \\
\hline & & Mean & SD & $\begin{array}{l}\text { Left } \\
\text { hand } \\
(\%)\end{array}$ & $\begin{array}{c}\text { Right } \\
\text { hand } \\
(\%)\end{array}$ \\
\hline Vibration-exposed & 131 & 39.3 & 10.6 & 24.8 & 31.2 \\
\hline Nonvibration-exposed & 47 & 44.7 & 9.4 & 6.5 & 8.9 \\
\hline Total & $\uparrow 78$ & 40.7 & 10.6 & 19.9 & 25.3 \\
\hline
\end{tabular}

increased to at least $28^{\circ} \mathrm{C}$ by the subject rubbing his hands or plunging his hands in warm water.

The tactilometer produced automatically increasing (appearance threshold) and decreasing (disappearance threshold) levels of vibration for seven discrete frequen$\operatorname{cies}(8,16,31.5,63,125,250$, and $500 \mathrm{~Hz}$ ) (table 2). The subject indicated with the other hand when the vibration was perceived by pressing a button on a hand switch. When the vibration decreased to a limit where he could no longer perceive it, he released the button. The thresholds reported are the average midpoints between the upper and lower limit of perception, expressed in decibels (reference $1 \mu \mathrm{m} \cdot \mathrm{s}^{-2}$ ).

The test frequency for the vibrameter was $100 \mathrm{~Hz}$. The examiner manually changed the amplitude of the vibration upwards by pressing a button on the apparatus, and the subject indicated when he could perceive the vibration (appearance threshold) by pressing a button on a hand switch. The thresholds were determined three times and an average was used. The vibrameter gave the thresholds in micrometers of peak-to-peak displacement.

Both devices controlled the pressure from the vibration exciter on the skin. The tactilometer provided a constant static pressure of $3.5 \mathrm{~N} \cdot \mathrm{cm}^{-2}$. The vibrameter used a pressure of approximately $4.8 \mathrm{~N} \cdot \mathrm{cm}^{-2}$ (from the weight of the stimulator). Both probes had plane and circular surfaces. The probe of the vibrameter and the tactilometer had a diameter of 13 and $6 \mathrm{~mm}$, respectively.

The methods, apparatus, and experimental procedure associated with these measurements has been described elsewhere in more detail $(4,11)$.

Table 2. Description of the two devices.

\begin{tabular}{lll}
\hline Characteristic & Vibrameter & Tactilometer \\
\hline Frequency & $100 \mathrm{~Hz}$ & $\begin{array}{l}8,16,31.5,63,125, \\
250,500 \mathrm{~Hz}\end{array}$ \\
Probe size & $13 \mathrm{~mm} \emptyset$ & $6 \mathrm{~mm} \emptyset$ \\
Apparatus set-up & Manually held & Fixed \\
Stimulation & Manual & Automatic \\
Measurement unit & Micrometers & Decibels $\left(\right.$ reference $\left.1 \mu \mathrm{m} \cdot \mathrm{s}^{-2}\right)$ \\
Contact force & $\sim 4.8 \mathrm{~N} \cdot \mathrm{cm}^{-2}$ & $3.5 \mathrm{~N} \cdot \mathrm{cm}^{-2}$ \\
Probe surround & None & None \\
\hline
\end{tabular}




\section{Data analysis}

The thresholds determined by the vibrameter were transformed to decibels (rel. $1 \mu \mathrm{m} \cdot \mathrm{s}^{-2}$ ) to be comparable with the values of the tactilometer.

The following four ways of combining the seven frequencies from the tactilogram have been suggested:

1. Use of the sensibility index (SI), calculated by dividing the area beneath the tactilogram (a curve of the seven thresholds) by the corresponding area of a reference population, both age-adjusted according to a suggested reference population (12).

2. Use of the low-frequency index (LFI), calculated as the mean of the measurements at 16 and $31.5 \mathrm{~Hz}$, the former converted to the level of $31.5 \mathrm{~Hz}$ as follows:

$$
\mathrm{LFI}=\left(\overline{\mathrm{X}}_{31.5 \mathrm{~Hz}} / \overline{\mathrm{X}}_{16 \mathrm{~Hz}}\right) \cdot \mathrm{X}_{16 \mathrm{~Hz}}+\mathrm{X}_{31.5 \mathrm{~Hz}}
$$

where $\bar{X}=$ the mean of the whole group and $x=$ the individual value.

3. Use of the high-frequency index (HFI), calculated as the mean of the measurements at $63,125,250$, and $500 \mathrm{~Hz}$, all converted to the level of $125 \mathrm{~Hz}$ as follows:

$$
\begin{aligned}
& \mathrm{HFI}=\left(\overline{\mathrm{X}}_{125 \mathrm{~Hz}} / \overline{\mathrm{X}}_{63 \mathrm{~Hz}}\right) \cdot \mathrm{x}_{63 \mathrm{~Hz}}+\mathrm{x}_{125 \mathrm{~Hz}}+\left(\overline{\mathrm{X}}_{125 \mathrm{~Hz}} /\right. \\
& \left.\overline{\mathrm{X}}_{250 \mathrm{~Hz}}\right) \cdot \mathrm{x}_{250 \mathrm{~Hz}}+\left(\overline{\mathrm{X}}_{125 \mathrm{~Hz}} / \overline{\mathrm{X}}_{500 \mathrm{~Hz}}\right) \cdot \mathrm{x}_{500 \mathrm{~Hz}}
\end{aligned}
$$

where $\bar{X}=$ the mean of the whole group and $x=$ the individual value (13).

4. Use of a simplified version of a grading system (GS) (14). Each subject's value was compared with the mean and standard deviation of the unexposed. The number of standard deviations $(0,1,2$ or $3 \mathrm{SD})$ by which the subject's value exceeded the mean was then summed for the seven frequencies, giving a range from 0 to 21 .
Comparisons were made for the discrete frequencies of the tactilometer, as well as for the four combinations.

Pearson correlations were used to evaluate the linear relationship between the vibrameter and tactilometer thresholds. It should be noted that the grading system is not normally distributed. Using rank correlations (Spearman) for these data did not, however, change the conclusion. Thresholds, indices, and grading values above the upper quartile were used as reduced vibrotactile perception for the purpose of assessing agreement between the highest thresholds. Kappa coefficients for agreement beyond chance have been given for these comparisons (15)

\section{Results}

The correlations between the vibrameter and the tactilometer were highest for the frequencies $63 \mathrm{~Hz}, 125 \mathrm{~Hz}$, and the high-frequency index (table 3). The correlations between the adjacent frequencies of the tactilometer were between 0.80 and 0.89 .

A plot of the differences between the two thresholds (tactilometer $125 \mathrm{~Hz}$ - vibrameter) and age did not show any pattern of larger differences with higher age. Neither did a plot of the differences and the mean of the two thresholds show a greater variability with a higher mean.

Comparisons were made between the thresholds above the upper quartile (table 3). The agreement between the vibrameter and the tactilometer $(63 \mathrm{~Hz})$, when the upper quartile was used as a limit, had a sensitivity of 0.56 and a specificity of 0.85 . These values correspond to a kappa value of 0.41 ( $95 \%$ confidence interval 0.26 0.57 ) for agreement beyond chance. The lowest agree-

Table 3. Correlation coefficients, sensitivity, and specificity between the thresholds above the upper quartile of the vibrameter and the

\begin{tabular}{|c|c|c|c|c|c|c|}
\hline \multirow[t]{3}{*}{ Tactilometer } & \multicolumn{6}{|c|}{ Vibrameter $(100 \mathrm{~Hz})$} \\
\hline & \multicolumn{2}{|c|}{ Correlation coefficient } & \multicolumn{2}{|c|}{ Left hand } & \multicolumn{2}{|c|}{ Right hand } \\
\hline & Left hand & Right hand & Sensitivity & Specificity & Sensitivity & Specificity \\
\hline \multicolumn{7}{|c|}{ Discrete frequencies $(\mathrm{Hz})$} \\
\hline $\begin{array}{l}8 \\
16 \\
31.5 \\
63 \\
125 \\
250 \\
500\end{array}$ & $\begin{array}{l}0.25 \\
0.35 \\
0.48 \\
0.54 \\
0.53 \\
0.47 \\
0.46\end{array}$ & $\begin{array}{l}0.21 \\
0.31 \\
0.47 \\
0.56 \\
0.59 \\
0.54 \\
0.49\end{array}$ & $\begin{array}{l}0.34 \\
0.42 \\
0.47 \\
0.56 \\
0.51 \\
0.49 \\
0.49\end{array}$ & $\begin{array}{l}0.78 \\
0.81 \\
0.82 \\
0.85 \\
0.84 \\
0.83 \\
0.83\end{array}$ & $\begin{array}{l}0.36 \\
0.43 \\
0.49 \\
0.55 \\
0.53 \\
0.49 \\
0.45\end{array}$ & $\begin{array}{l}0.77 \\
0.79 \\
0.82 \\
0.84 \\
0.83 \\
0.82 \\
0.80\end{array}$ \\
\hline \multicolumn{7}{|c|}{ Different indices } \\
\hline $\begin{array}{l}\mathrm{SI} \\
\mathrm{LFI} \\
\mathrm{HFI}\end{array}$ & $\begin{array}{l}0.43 \\
0.44 \\
0.53\end{array}$ & $\begin{array}{l}0.44 \\
0.41 \\
0.57\end{array}$ & $\begin{array}{l}0.50 \\
0.49 \\
0.49\end{array}$ & $\begin{array}{l}0.83 \\
0.83 \\
0.83\end{array}$ & $\begin{array}{l}0.47 \\
0.49 \\
0.51\end{array}$ & $\begin{array}{l}0.81 \\
0.82 \\
0.82\end{array}$ \\
\hline \multicolumn{7}{|c|}{ Grading system } \\
\hline GS & 0.46 & 0.53 & 0.50 & 0.83 & 0.56 & 0.84 \\
\hline
\end{tabular}
discrete frequencies, the high and low frequency indices (HFI and LFI), the sensibility index (SI), and the grading system (GS) of the tactiometer. 
ment was between the vibrameter and $8 \mathrm{~Hz}$ for the tactilometer with a kappa value of $0.12(95 \%$ confidence interval $-0.04-0.28$ ).

In a scatter plot of the right-hand thresholds (tactilometer $125 \mathrm{~Hz}$ and vibrameter), the subjects who reported symptoms were distributed over the whole scale of thresholds for both instruments (figure 1). All told, $44 \%$ of the 43 subjects reporting sensorineural symptoms of the right hand and $21 \%$ of the 135 subjects without symptoms had a threshold above the upper quartile with the tactilometer $(63 \mathrm{~Hz}$ and HFI). The corresponding percentages for the vibrameter were $40 \%$ for the subjects with symptoms and $22 \%$ for subjects without symptoms. These values correspond for to a sensitivity and specificity of 0.44 and 0.79 , respectively, for the tactilometer and 0.40 and 0.78 , respectively, for the vibrameter. The right and left hands showed almost identical results. The proportion of vibration-exposed persons among the subjects who belonged above the upper quartile varied between $67 \%$ and $89 \%$ (figure 2). The sensitivity and specificity for classifying the subjects as exposed and unexposed was 0.89 and 0.96 , respectively, for the vibrameter and 0.81 and 0.93 , respectively, for the tactilometer $(63 \mathrm{~Hz})$ for the right hand.

\section{Discussion}

The correlations between the thresholds from the vibrameter and the tactilometer were, even at the closest frequencies ( $r \leq 0.59)$, not as high as the correlation between one frequency of the tactilometer and the next

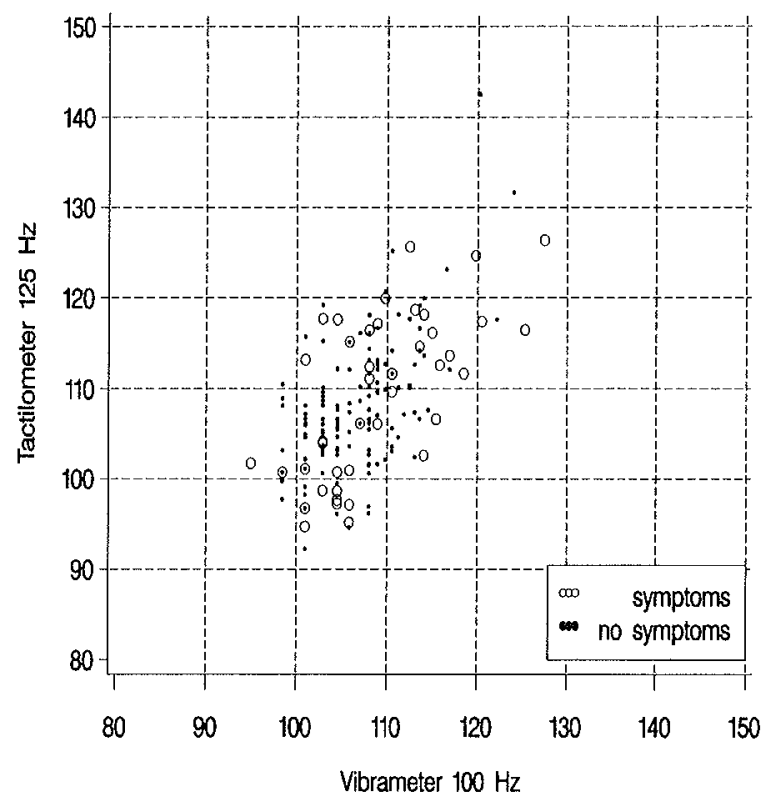

Figure 1. Scatter plot of right-hand thresholds for the tactilometer $(125 \mathrm{~Hz})$ and the vibrameter.

$(r \geq 0.80)$. The agreement between thresholds above the upper quartile for each device was only slightly higher than what would be expected by chance (kappa $\leq 0.41$ ). Landis \& Koch (15) have characterized kappa values below 0.4 for representing poor agreement and values between 0.4 and 0.75 for representing fair to good agreement.

Other authors have compared results of different devices and found that the vibrotactile perception thresh-

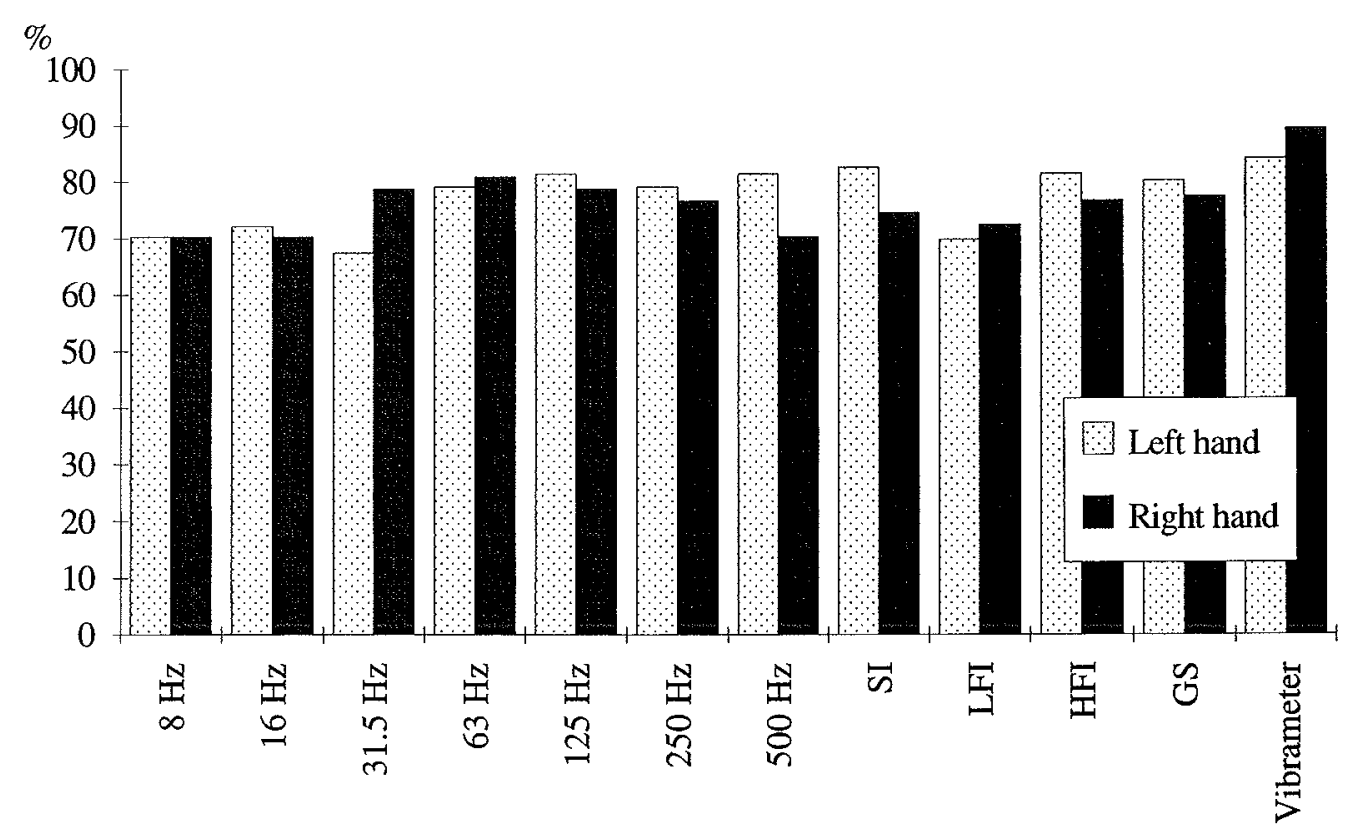

Figure 2. Proportion of vibration-exposed persons among the subjects with reduced vibrotactile perception (thresholds above the upper quartile). ( $\mathrm{SI}=$ sensibility index, $\mathrm{LFI}=$ low frequency index, $\mathrm{HFI}=$ high frequency index, $\mathrm{GS}=$ grading system) 
olds vary from one piece of equipment to another (16, 17). Rosén compared the vibrameter with a sensorineural staging based on the tactilometer and found a weak correlation only (18).

As many influencing factors as possible were standardized to obtain comparable results for the two devices. The finger temperature was controlled and adjusted before each of the measuring procedures, the position of the probe on the finger was standardized as exactly as possible, and the surroundings (noise or other disturbances) were identical. There were, however, other factors that could have influenced the measurements and could therefore be possible explanations for the discrepancies in the results.

It has been suggested that vibration perception thresholds at low $(<5 \mathrm{~Hz})$, medium $(<50 \mathrm{~Hz})$, and high frequencies $(>50 \mathrm{~Hz}$ ) are mediated by activity from different types of mechanoreceptors in the skin (19). The vibrameter was therefore not expected to show strong agreement with the highest and lowest frequencies of the tactilometer. But even at the frequencies closest to $100 \mathrm{~Hz}$ the correlation was markedly low $(\mathrm{r} \leq 0.59)$ when compared with correlation coefficients in the range of $0.80-0.89$ between adjacent frequencies of the tactilometer.

The tactilometer registered both the appearance and disappearance thresholds, and the average midpoint between the upper and lower limit of perception was used. For the vibrameter only the appearance threshold was registered. It is possible to register the disappearance threshold as well, but the appearance threshold is the most commonly used in practice for the Somedic vibrameter. Analyses were also performed with appearance thresholds from the tactilometer (ie, an average of the upper threshold limit); the agreement was, however, approximately the same.

The experimental procedure of the tactilometer can possibly be regarded as more difficult for the subject, since the vibration level is increased and decreased several times at each frequency. An advantage of this procedure is that it is impossible for the subject to guess when he is supposed to feel anything if he is eager to produce a good and reproducible test result. If a person is guessing, he would soon be out of phase, and the test protocol would reveal it. The vibrameter does not have this assurance. However, the examiner judged the subjects as calm, relaxed, and interested in following the instructions given.

The tactilometer was fixed, and the finger was positioned from below with the arm resting on a support. The vibrameter was manually held on the finger by the examiner. It is possible that the vibrameter probe was not kept as stable as the tactilometer, even if the pressure was kept constant. The effects of an inclination of the probe have not been studied but could make the pressure differ within the probe area or stimulate a different part of the finger than was intended. Such effects probably do not have a great impact on the measurements but could explain part of the variation.

The vibrameter used a probe which was $13 \mathrm{~mm}$ in diameter, and the tactilometer probe was $6 \mathrm{~mm}$ in diameter. The effect of the contactor size has been studied (7). It is possible that two probes which cover different areas of the receptors can be one reason for the discrepancies in the results.

Harada concluded that a larger contact force led to lower thresholds at 125,250 , and $500 \mathrm{~Hz}$ in five male subjects between the ages of 23 and 28 years (6). The differences between the contact forces of 1 and $3 \mathrm{~N}$ varied between 2 and $6 \mathrm{~dB}$. The tactilometer used a pressure of $3.5 \mathrm{~N} \cdot \mathrm{cm}^{-2}$, whereas that of the vibrameter was approximately $4.8 \mathrm{~N} \cdot \mathrm{cm}^{-2}$. The difference is so small that it could not have resulted in large differences between the thresholds.

One examiner for each device conducted the measurements for all the subjects. It has been shown that results can depend on different examiners (20). It is possible that this experimental factor can have influenced the measurements.

Several authors have discussed the problems involved with a large inter- and intraindividual variability in VPT measurements. Aaserud et al (21) reported a high interand intraindividual variation for 12 healthy subjects at frequencies of $25,50,100$, and $250 \mathrm{~Hz}$. Fagius \& Wahren (22) reported a highly significant intraindividual variation for 13 healthy subjects and for 27 patients with polyneuropathy at $100 \mathrm{~Hz}$. Löfvenberg \& Johansson (23) showed that $27 \%, 27 \%$, and $67 \%$ of the variance in the measurements was explained by the subject factor for the frequencies 2,25 , and $200 \mathrm{~Hz}$, respectively, when 11 healthy subjects (17-36 years of age) were used. Sallé \& Verberk (17) reported an intraindividual variation of $30-50 \%$ for 12 healthy subjects, and Halonen (11) found a $100 \%$ variation for one subject measured on 20 days, both studies done at $100 \mathrm{~Hz}$. Aatola et al (24) reported repeatable results but a high interindividual variation.

Test-retest correlations have been reported by several authors to be in the range of $0.71-0.87(17,25,26)$. These results agreed with the correlation between two adjacent frequencies of the tactilometer in our study. The difference between the results of the two devices can probably partly be explained by a large intraindividual variation in each of the devices. The physical parameters mentioned, such as the size of the probe, probably causes mainly systematic differences that should not influence the correlations.

The two devices seemed to have similar relations to exposure and sensorineural hand symptoms. The results can be compared with the results of Virokannas (13), 
who showed a sensitivity of $68 \%$ (with a constant specificity of $90 \%$ ) when using the high-frequency index to discriminate between 77 exposed and 70 unexposed persons. Ekenvall et al (2) reported a sensitivity of 32\% and a specificity of $92 \%$ when discriminating sensorineural stages 2 and 3 from stages 0 and 1. Lundborg et al (27) showed a sensitivity of $83 \%$ and a specificity of $46 \%$ when the outcome of a neurophysiological test was used as the standard. These results agree to some extent with the results from the present study; however, the percentages in this study should not be interpreted as real sensitivities since no confounding was taken into account. The exposure categories used in this study should not be considered as a gold standard. If perfect agreement is to be expected, a more-detailed exposure assessment would probably be needed.

The high interindividual variation in the results makes it difficult to set a division point with both high sensitivity and high specificity for agreement with a standard. Different division points have been suggested for the two devices, but they have been constructed with different restrictions on the specificity. When the number of measurements above the limit for normal perception is allowed to vary for the two devices, it is difficult to evaluate the agreement. In order not to let the division points influence the comparisons, the upper quartile was used to classify the thresholds as extreme or normal. For future use of the devices, there is a need to find and evaluate different division points for different purposes (eg, screening and diagnosis).

Some authors have reported high sensitivities without specifying the specificity $(3,12,28)$.

Other authors have shown that mean perception thresholds are useful when discriminating between vibration-exposed and vibration-unexposed groups, as well as between groups with and without symptoms $(2,13$, $18,29)$. The use of group means to assess the usefulness of the measurements for the purpose of individual diagnosis or screening is questionable, since a few extreme measurements in the exposed group can be enough to make the means differ significantly. For individual diagnosis or screening a division point has to be specified, and both sensitivity and specificity need to be reported.

The magnitude of agreement between the thresholds, exposure, and symptoms agreed with the sensitivities and specificities reported by other authors.

Several authors have concluded that a large interindividual variability restricts the usefulness to comparisons of groups or as a complement to other diagnostic techniques $(18,24,30)$. Fagius \& Wahren (22) concluded that the large intraindividual variation restricts the usefulness in longitudinal studies of individual subjects.

This study supports the conclusion that vibrotactile perception thresholds involve a large amount of interand intraindividual variability that restricts the useful- ness for screening and diagnosis on an individual basis. They can still be valuable when discriminating between groups or as a complement to other diagnostic techniques. Neither of the two devices was shown to be superior in this study when the results were compared with the occurrence of symptoms and exposure.

\section{Acknowledgments}

The study was supported by grants from the Swedish Work Environment Fund (91-1640).

The authors would like to thank the many individuals whose support made this study possible, particularly Ms Asta Lindmark and Ms Astrid Fägerstrand, who conducted the vibrotactile perception threshold measurements.

\section{References}

1. Brammer AJ, Taylor W, Piercy JE. Assessing the severity of the neurological component of the hand-arm vibration syndrome. Scand J Work Environ Health 1986;12:428-31.

2. Ekenvall L, Gemne G, Tegner R. Correspondence between neurological symptoms and outcome of quantitative sensory testing in the hand-arm vibration syndrome. Br J Ind Med $1989 ; 46: 570-4$.

3. Lundborg G, Sollerman C, Strömberg T, Pyykkö I, Rosén B. A new principle for assessing vibrotactile sense in vibrationinduced neuropathy. Scand J Work Environ Health 1987;13: $375-9$

4. Lundström R, Strömberg T, Lundborg G. Vibrotactile perception threshold measurements for diagnosis of sensory neuropathy: description of a reference population. Int Arch Occup Environ Health 1992;64:201-7.

5. Brammer AJ, Taylor W, Lundborg G. Sensorineural stages of the hand-arm vibration syndrome. Scand J Work Environ Health 1987;13:279-83.

6. Harada N, Griffin MJ. Factors influencing vibration sense thresholds used to assess occupational exposures to hand transmitted vibration. Br J Ind Med 1991;48:185 -92.

7. Verillo R'T. Effect of contactor area on the vibrotactile threshold. J Acoust Soc Am 1963;35(12):1962—6.

8. Gerr F, Letz R. Vibrotactile threshold testing in occupational health: a review of current issues and limitations. Environ Res 1993;60:145-59.

9. Goldberg JM, Lindblom U. Standardised method of determining vibratory perception thresholds for diagnosis and screening in neurological investigation. J Neurol Neurosurg Psychiatry 1979;42:793-803.

10. Verberk MM, Sallé HJA, Kempers O. Vibratory and tactile sense of the fingers after working with sanders. Int Arch Occup Environ Health 1985;56:217-23.

11. Halonen P. Quantitative vibration perception thresholds in healthy subjects of working age. Eur J Appl Physiol 1986;54: $647-55$.

12. Lundborg G, Dahlin LB, Lundström R, Necking LE, Ström- 
berg $\mathrm{T}$. Vibrotactile function of the hand in compression and vibration-induced neuropathy: sensibility index - a new measure. Scand J Plast Reconstr Hand Surg 1992;26:275-79.

13. Virokannas $\mathrm{H}$. Vibration perception thresholds in workers exposed to vibration. Int Arch Occup Environ Health 1992; 64:377-82.

14. Lundström R, Hagberg M, Johansson K, Lindmark A, Nilsson T. Handens känselfunktion hos plåtslagare och montörer utsatta för vibrationer [Sensorineural disturbances in vibrationexposed hands among platers and assemblers]. Stocholm: Arbetarskyddsverket, 1991:1—24. Arbete och hälsa 42:1991. Summary in English.

15. Landis RJ, Koch GG. The measurement of observer agreement for categorical data. Biometrics 1977;33:159-74.

16. Maeda S, Griffin MJ. A comparison of vibrotactile thresholds on the finger obtained with different equipment. Ergonomics 1994;37(8):1391— 406.

17. Sallé HJA, Verberk MM. Comparison of five methods for measurement of vibratory perception. Int Arch Occup Environ Health 1984:53:303-9.

18. Rosén I, Strömberg T, Lundborg G. Neurophysiological investigation of hands damaged by vibration: comparison with idiopathic carpal tunnel syndrome. Scand J Plast Reconstr Hand Surg 1993;27:209-16.

19. Lundström RJI. Responses of mechanoreceptive afferent units in the glabrous skin of the human hand to vibration. Scand J Work Environ Health 1986;12:413-6.

20. Gerr F, Letz R. Covariates of human peripheral nerve function: II. vibrotactile and thermal thresholds. Neurotoxicol Teratol 1994;16(1):105-12.

21. Aaserud O, Juntunen J, Matikainen E. Vibration sensitivity thresholds: methodological considerations. Acta Neurol Scand 1990;82:277-83.

22. Fagius J, Wahren LK. Variability of sensory threshold determination in clinical use. J Neurol Sci 1981;51:11—27.

23. Löfvenberg J, Johansson RS. Regional differences and interindividual variability in sensitivity to vibration in the glabrous skin of the human hand. Brain Res 1984:301:65-72.

24. Aatola S, Färkkilä M, Pyykkö I, Korhonen O, Starck J. Measuring method for vibration perception threshold of fingers and its application to vibration exposed workers. Int Arch Occup Environ Health 1990;62:239 — 42 .

25. Grunert BK, Wertsch JJ, Matloub HS, McCallum-Burke S. Reliability of sensory threshold measurement using a digital vibrogram. J Occup Med 1990;32(2):100-2.

26. Gerr FE, Letz R. Reliability of a widely used test of peripheral cutaneous vibration sensitivity and a comparison of two testing protocols. Br J Ind Med 1988;45:635-9.

27. Lundborg G, Lie-Stenström A-K, Sollerman C, Strömberg T, Pyykkö I. Digital vibrogram: a new diagnostic tool for sensory testing in compression neuropathy. J Hand Surg 1986;11A: 693-9.

28. Brammer AJ, Piercy JE, Auger PL, Nohara S. Tactile perception in hands occupationally exposed to vibration. $\mathbf{J}$ Hand Surg 1987;12A:870-5.

29. Lundström R. Effects of local vibration transmitted from ultrasonic devices on vibrotactile perception in the hands of therapists. Ergonomics 1985;28(5):793-803.

30. Pyykkö I. Clinical aspects of the hand-arm vibration syndrome. Scand J Work Environ Health 1986;12:439-47.

Received for publication 20 March 1995 